\title{
Le système Guénon : une innovation technique pour l'amélioration de la sélection des vaches laitières (années 1830-années 1900)
}

\author{
The Guénon System: A Technological Innovation for the Improvement of \\ Dairy Cow Selection (1830s-1900s)
}

\author{
Fabien Knittel ${ }^{1}$ \\ ${ }^{1}$ Centre Lucien Febvre, Université de Bourgogne-Franche-Comté (UFC), France, fabien.knittel@univ-fcomte.fr
}

\begin{abstract}
RÉSUMÉ. Cet article a pour objet principal l'analyse de la trajectoire originale d'un marchand de bestiaux bordelais, François Guénon (1796-1855), concepteur d'un système innovant destiné à améliorer la sélection des meilleures vaches laitières, à la fin des années 1830 (décrit dans son Traité des vaches laitières, publié pour la première fois à Bordeaux en 1838). Son système empirique de sélection des " bonnes " laitières rencontre un réel succès auprès des éleveurs ainsi que des vétérinaires ou de certains agronomes. Cependant, ce succès initial est discuté par la suite, notamment à partir les années 1860, par certains agronomes, comme Eugène Tisserant (1818-1888), alors professeur d'hygiène à l'école vétérinaire de Lyon, qui ne le considèrent pas comme sûr et le jugent insuffisamment fondé scientifiquement. Or, ces remarques critiques n'entament en rien le recours au système Guénon par de nombreux éleveurs et n'empêche pas non plus son enseignement au sein de certains établissements techniques agricoles au début du XX ${ }^{e}$ siècle.

ABSTRACT. This paper principally aims to examine the original career path of François Guénon, a cattle merchant from Bordeaux. In the late 1830s, he set up a pioneering system the purpose of which was to improve the selection of the most effective dairy cows. The system was dealt with in the article Traité des vaches laitières, first published in 1838. The empirical process which aimed to pick the best dairy cows was successfully implemented. However, from the 1860s onwards, this initial success was debated among agronomists like Eugène Tisserant (1818-1888), a then Professor of hygiene at the veterinary school of Lyon, who dismissed his work as scientifically groundless. Nevertheless, these critical comments neither weakened the efficiency and the use of the Guénon system nor prevented its teaching within certain farming schools at the beginning of the $20^{\text {th }}$ century.

MOTS-CLÉS. Innovation, techniques, vaches laitières, XIX $X^{e}$ siècle, François Guénon, agronomie, histoire.

KEYWORDS. Innovation, Technology, Dairy Cows, $19^{\text {th }}$ century, François Guénon, Agronomy, History.
\end{abstract}

\section{Introduction}

François Guénon (1796-1855) est un marchand de bestiaux bordelais qui a élaboré un système d'identification des vaches laitières durant les années 1830. Son objectif est de fournir aux éleveurs laitiers une technique efficace et systématique leur permettant d'identifier une future bonne laitière. C'est une démarche originale qui s'inscrit dans un double contexte : celui du renforcement de la spécialisation des exploitations agricoles, dont certaines dans l'élevage laitier, surtout dans les montagnes [ANT 16 ; MAD 06] ; celui du développement de la zootechnie et des principes de la sélection animale dans le but d'améliorer les races d'élevage [VIS 02].

Guénon est avant tout un praticien dont le savoir est principalement empirique, c'est-à-dire un ensemble de connaissances qui relèvent de l'expérience sensu stricto [RUT 04, p. 412]. Son système est élaboré puis soumis à la communauté agricole et savante, par l'intermédiaire du comice de Bordeaux et des sociétés d'agriculture, en plein essor sous la Monarchie de Juillet, à partir uniquement de ses observations quotidiennes. Il ne rejette pas les savoirs agronomiques et vétérinaires mais son métier de marchand de bovins lui apporte les ressources, fondée sur l'observation régulière des vaches principalement, qui fondent son système d'identification dont il espère, in fine, une reconnaissance ensuite par les instances de légitimation que sont les comices et les sociétés savantes agricoles. Toute l'histoire du système Guénon est marquée par cette tension entre empirisme et connaissances scientifiques des agronomes et des vétérinaires. 
Cet article a pour objet principal l'analyse de la trajectoire originale de Guénon, individu obscur passé soudainement à la lumière grâce à un procédé qu'il présente lui-même comme «révolutionnaire » et surtout, infaillible. Sa technique est alors considérée comme innovante. Accepté comme tel dans un premier temps, tant par les agronomes que par les empiriques, le système Guénon est ensuite discuté et critiqué par certains agronomes, notamment à partir des années 1860 par Eugène Tisserant (1818-1888), professeur d'hygiène à l'école vétérinaire de Lyon. Ce critique du système Guénon ne doit pas être confondu avec Eugène Tisserand (1830-1925), sommité de l'agronomie, à partir des années 1870 acteur clé du développement de l'agronomie, Inspecteur général de l'agriculture en 1871, membre de l'Académie d'agriculture dès janvier 1874, directeur de l'agriculture au Ministère de 1878 à 1896 et, enfin, membre de l'Académie des sciences en 1911 [BOU 98, p. 199-213].

Pour étudier l'histoire de cette innovation, les sources utilisées sont principalement le Traité des vaches laitières rédigé par Guénon et publié une première fois en 1838 ainsi que ses rééditions, y compris posthumes. Pour aborder les critiques formulées au sujet de la technique proposée par Guénon, la littérature agronomique et vétérinaire a été utilisée, notamment certains écrits de Jean-Henri Magne ou encore d'Eugène Tisserant. Des périodiques de sociétés savantes agricoles ont aussi été utilisés comme les Annales agricoles et littéraires de Dordogne par exemple.

L'élevage est rarement considéré comme un domaine important de l'économie rurale au XIX ${ }^{\mathrm{e}}$ siècle. Par exemple, Adrien de Gasparin, dans son Cours d'agriculture de 1843, se demande si «l'éducation des animaux doit-elle être regardée comme partie intégrante de la science agricole ? » Il répond négativement en faisant une distinction nette entre agriculture et zootechnie [GAS 43]. Certains agronomes consacrent cependant de longues pages à l'élevage qui devient, surtout dans la seconde moitié du siècle, un domaine important à relier à la spécialisation progressive des exploitations agricole, notamment en montagne pour l'élevage bovin-lait [BOI 77]. Le point de vue des vétérinaires est différent certes mais leurs textes, s'ils traitent beaucoup des épizooties, abordent aussi les aspects zootechniques en lien avec les techniques d'élevage dans le cadre plus large de l'économie rurale [VAL $15]$.

Il s'agit donc de comprendre une trajectoire d'innovation originale entre acceptation et rejet en même temps que de s'interroger sur les déterminants sociaux de l'innovation et la place d'un innovateur « ordinaire », c'est-à-dire un empirique soucieux de diffuser ses inventions techniques dans les mondes ruraux, tel que Guénon, issu du peuple, dont le système est contesté par les «autorités scientifiques » agronomiques et vétérinaires. C'est principalement cette question des innovations provenant des «petites gens de la terre » qui nous intéresse ici. Cette problématique est complétée par une réflexion sur les enjeux des controverses dans la légitimation et la structuration disciplinaires des sciences (ici, l'agronomie, la zootechnie et la médecine vétérinaire) au cours du XIX ${ }^{\mathrm{e}}$ et au début du $\mathrm{XX}^{\mathrm{e}}$ siècle, pour lesquelles l'exemple Guénon est éclairant.

Pour aborder ces questions nous commençons par décrire le système innovant proposé par Guénon en en donnant les principales caractéristiques et en précisant les modalités de sa diffusion. Dans un second temps, nous montrons que le système Guénon est une technique discutée et critiquée par les agronomes mais que Guénon défend ses idées et que certains empiriques le soutiennent. Enfin, nous finissons sur un paradoxe : la techniques Guénon, à la fin du XIX ${ }^{\mathrm{e}}$ siècle, est toujours critiquée avec virulence et semble disparaître progressivement alors qu'elle est toujours enseignée dans certaines écoles techniques agricoles comme l'Ecole nationale des industries laitières (ENIL) de Mamirolle, dans le département du Doubs, au début du $\mathrm{XX}^{\mathrm{e}}$ siècle.

\section{Un système innovant}

\subsection{Les caractéristiques du système Guénon}

Le système Guénon est élaboré par un marchand de vaches de la région bordelaise, François Guénon, inconnu jusqu'alors. Il est identifié dans un premier temps comme jardinier, terme vague à 
l'époque, puis, d'après les Annales agricoles et littéraires de Dordogne, comme marchand de vaches à Libourne dans le département de la Gironde. Guénon est donc un empirique, relativement anonyme, sans réelle formation technique ou scientifique.

Guénon présente son système pour la première fois en juillet 1837 lors du comice agricole de Bordeaux [KNI 20]. Il s'agit d'une technique visuelle permettant de reconnaître, et de sélectionner, les meilleures vaches laitières. Récompensé par une médaille d'or lors du comice de Bordeaux en 1837, Guénon fait paraître un Traité des vaches laitières dès l'année suivante [GUE 38]. Publié pour la première fois en 1838 à Bordeaux, l'ouvrage connaît rapidement plusieurs rééditions, indice de son succès. Le Traité de Guénon est, dès 1861, réédité pour la quatrième fois.

Concrètement, la technique de Guénon consiste à relever les marques «distinctives de chaque animal (...) visibles sur chaque vache, à la partie postérieure, entre le pis et la vulve. Ce sont des espèces d'écussons de différentes formes et grandeurs, et formés par des lignes de contre poil tantôt verticales, tantôt transversales, dont les variétés indiquent la classe et l'ordre auxquels appartient l'individu » [GUE 38, p. 35]. Ensuite, Guénon explique qu'avec sa technique les éleveurs sont en mesure d'identifier « (...) des marques positives, non équivoques, infaillibles, auxquelles on peut juger une jeune vache dès l'âge le plus tendre et l'apprécier sans erreur ; (...) ces marques, que la nature ellemême a imprimées à chaque individu, selon sa catégorie, sont visibles à l'œil » [GUE 38, p. 11]. Guénon propose donc une taxinomie des vaches laitières à partir de la forme des écussons de celles-ci, qui correspond d'après lui à des signes probants de la qualité de la lactation des vaches. Il revendique toutefois sa qualité «d'empirique » en instant sur les tâtonnements de sa démarche dans l'élaboration de sa technique d'identification. Fondée sur l'observation avant tout, la technique Guénon est cependant une manière de faire qui se rapproche d'une pratique de type expérimental. Il indique qu'il lui a « fallu méditer, calculer, combiner, rectifier des erreurs, établir des classes, des ordres, dans chaque classe, des degrés dans chaque ordre... » [GUE 38, p. 17-18]. Les marques identifiées par Guénon correspondent à des écussons visibles sur la partie postérieure des vaches « entre le pis et la vulve » de l'animal [GUE 38, p. 35]. Ces écussons, le vétérinaire Jean-Henri Magne (1804-1885) [LIJ 06], dans son ouvrage Choix des vaches laitières, publié pour la première fois en 1853 et réédité dès 1857, les définit comme une plaque de poils qui recouvre pis et périnée des vaches, de forme et grandeur variées [MAG 57, p. 6]. Les lignes de poils sont soit verticales soit transversales. À partir de ces critères Guénon élabore un classement des différents types de vaches laitières, croisant les formes d'écussons avec la taille des vaches pour aboutir à trois catégories principales : grandes, moyennes, petites ; une fois tous les critères sériés et croisés Guénon obtient huit classes réparties en trois «ordres » principaux pour reprendre ses propres termes [GUE 38]. Au final, il obtient huit classes de vaches, chaque classe étant subdivisée en grande, moyenne ou basse taille, elles-mêmes subdivisées en huit ordres différents. Son Traité des vaches laitières est richement illustré par des gravures représentant chaque type de vache ordonné par ordre au sein de chaque classe [GUE 38, planches hors texte]. Sur chaque gravure les formes des écussons sont indiquées par des lettres qui servent de points de repère, facilitant la «lecture » des écussons et la compréhension de la technique par ceux qui souhaitent s'y initier.

Cette technique d'identification convainc de nombreux éleveurs et marchands de bestiaux qui annoncent l'utiliser et considèrent que ce moyen de sélection est efficace. La technique Guénon est donc largement diffusée à partir de la fin des années 1830.

\subsection{Une technique largement diffusée}

Le système Guénon de sélection des «bonnes » laitières rencontre un réel succès auprès des éleveurs mais aussi auprès de certains vétérinaires et agronomes. La pratique empirique proposée par Guénon est reconnue par certains savants ce qui lui confère une légitimité et une notoriété. Il est d'ailleurs récompensé par les membres de la société d'agriculture d'Aurillac et ceux du comice de Bordeaux. Bien que ni agronome ni vétérinaire, Guénon est invité à présenter ses travaux à l'Académie de Bordeaux et à la Société d'agriculture d'Aurillac. Ces sociétés savantes jouent leur rôle de « tribunal de la science » [HIL 00 ; ROC 78] en validant la technique proposée par Guénon. Ce dernier, grâce à ces récompenses, accède à la notoriété dans les mondes de l'élevage bovin laitier. 
La mise par écrit par Guénon de ses idées et démarches dans un Traité des vaches laitières, est l'occasion pour lui de réécrire son histoire et de donner au processus de découverte une linéarité qui donne aux lecteurs une impression de simplicité, lui apportant l'apparence d'une péripétie heureuse aux enchaînements logiques : « un jour par passe-temps je m'amusais à gratter les épis que formait le rebours du poil au derrière de ma pauvre compagne [la vache familiale qu'il garde au pâturage], je m'aperçus qu'il s'en détachait une espèce de son assez abondant. Cette particularité attira mon attention; je me rappelai avoir ouï dire à un de mes ascendants que les vaches devaient avoir des signes extérieurs auxquels on pût reconnaître leurs qualités et leurs défauts... » [GUE 38, p. 15]. Rien de très original à cela, c'est un procédé rhétorique assez commun dans l'histoire des découvertes scientifiques et techniques, comme l'ont montré, dans un tout autre contexte, Bruno Latour et Steeve Woolgar dans La vie de laboratoire [LAT 79].

Grâce aux essais organisés par l'Académie de Bordeaux et aux divers textes rédigés à propos de la technique Guénon, celle-ci est rapidement et largement diffusée. Les académiciens bordelais font effectuer, dès 1828, des comparaisons entre troupeaux de vaches pour vérifier la fiabilité des propositions de Guénon : «des épreuves ont été faites avec le soin et les précautions nécessaires pour prévenir toute collusion; elles ont eu lieu sur trois troupeaux comptant en tout trente têtes...» (Rapport lu en séance le 5 juin 1828). De nombreux articles du Journal d'Agriculture Pratique abordent la technique mise au point par Guénon et en discutent le bien-fondé jusqu'aux années 1850 [DEN 98, p. 226-227]. Autre exemple, dans son ouvrage Les vaches laitières datant de 1895, le vétérinaire Emile Thierry (1839-1907) consacre encore près de dix pages au système Guénon [THI 95, p. 136-145].

Après la première édition en 1838, on compte près de vingt éditions du Traité des vaches laitières entre 1851 et 1899. Les traductions et rééditions d'un ouvrage sont certes un indicateur imparfait de la diffusion réelle de celui-ci mais donnent cependant une vue globale de sa propagation. A l'échelle européenne le Traité des vaches laitières a reçu un accueil favorable puisqu'il a été traduit en plusieurs langues, dont l'Anglais, l'Italien, l'Allemand et le Néerlandais. Par exemple, en Italie le Trattato delle vacche lattaje est publié en 1849. Dans l'espace germanique, on compte quatre éditions en langue allemande entre 1843 et 1845 . On peut mesurer aussi le succès, donc la diffusion large, des idées contenues dans le Traité des vaches laitières à la réédition posthume de l'œuvre. La quatrième édition française, en 1861, est réalisée six ans après la mort de Guénon ; preuve que sa technique lui survit et qu'elle retient encore l'intérêt dans les campagnes et les cabinets des agronomes après la mort de son inventeur.

Dans le cas présent l'origine de l'innovation est paysanne, ce qui est rare au XIX ${ }^{\mathrm{e}}$ siècle. Toutefois, les innovations paysannes, si elles sont, comme c'est le cas ici avec le système Guénon, validées par les sociétés savantes agricoles, sont largement diffusées grâce aux bulletins des académies d'agriculture de province et aux publications des comices agricoles [VIV 15]. Les petites gens de la terre sont donc parfois à l'origine d'innovations techniques d'ampleur, reprises et discutées ensuite par les savants qui en assurent la diffusion et la notoriété dans leurs réseaux. Certaines innovations issues des paysans sont médiatisées par le vecteur des publications des sociétés savantes qui en permettent la diffusion, la réception et, donc, la critique. C'est un exemple de la grande complexité des mécanismes de l'innovation technique en agriculture.

Cependant cette large diffusion, si elle permet la réception de l'innovation et entraine des éloges, n’évite pas les critiques négatives.

\section{Une innovation discutée dans un second temps}

\subsection{Des agronomes critiques}

Dès la parution du Traité des vaches laitières à la fin des années 1830, les critiques et réticences s'expriment parallèlement aux louanges. La technique proposée par Guénon ne laisse pas le milieu de l'élevage indifférent, son Traité est donc réédité plusieurs fois tout en étant commenté par de 
nombreux auteurs, tant agronomes que vétérinaires. D'autant plus que le contexte de la fin des années 1830 est aussi celui de la naissance de la zootechnie d'où les nombreuses analyses et critiques adressées à Guénon [VIS 02]. Jean-Henri Magne, vétérinaire et professeur d'agriculture et d'hygiène à l'école vétérinaire d'Alfort, remarque dans l'introduction de son ouvrage, Choix des vaches laitières, que « la nécessité de bien choisir (...) les vaches laitières (...) n'avait jamais attiré l'attention comme elle le fait depuis que M. Guénon a avancé et soutenu qu'on pouvait, avec facilité, apprécier exactement les qualités des vaches quant à la quantité, à la qualité du lait qu'elles peuvent donner, et à la durée de cette production » [MAG 57, p. 5-6]. Toutefois, Jean-Henri Magne ajoute aussitôt que « nous savons, par expérience (...), combien il est difficile d'apprécier les écussons d'après ce qu'en disent les auteurs qui les ont pris pour base de leur système » [MAG 57, p. 7]. Il reproche à Guénon la complexité de sa taxonomie et ajoute qu'il a «plusieurs fois conseillé à M. Guénon de simplifier son système... » [MAG 57, p. 7]. Le vétérinaire Magne, critique sévère, n’en reconnaît pas moins le rôle de précurseur joué par Guénon.

Magne ne condamne pas le procédé d'identification des bonnes laitières grâce à l'observation des écussons mais affirme que ceux qui ont proposés ce type de système ne se sont pas fait clairement comprendre, ce qui lui permet de justifier son propre ouvrage consacré au choix des vaches laitières. Si des signes existent pour identifier une bonne vache laitière, les seuls écussons « ne saurai[en]t suffire pour choisir avec certitude » [MAG 57, p. 7]. Les comices agricoles achètent et diffusent la littérature zootechnique afin de la mettre à disposition de leurs membres (à moindre coût), propriétaires, grands et moyens, et paysans aisés. La critique du système Guénon par Magne est donc aussi largement diffusée que le Traité des vaches laitières de Guénon lui-même.

Les critiques du système Guénon ne se résument cependant pas à une simple opposition entre empirique et vétérinaire. Elles reflètent, en partie, les luttes d'influences qui se jouent dans le cadre de la structuration de la zootechnie comme discipline scientifique à part entière et aussi, en partie, celles qui agitent la médecine vétérinaire. Aussi certains empiriques rejoignent-ils les considérations des vétérinaires. Par exemple, Lodieu, agriculteur et auteur d'ouvrages d'économie rurale [LOD 56], reproche aussi à Guénon la complexité de son système.

Le succès initial du système Guénon est donc discuté et il l'est de plus en plus à partir les années 1860. Certains agronomes, comme Eugène Tisserant, professeur d'hygiène à l'école vétérinaire de Lyon, ne le considèrent pas comme sûr et le jugent insuffisamment fondé scientifiquement. Dans son Guide (...) des vaches laitières, publié en 1858, Tisserant développe ses critiques et met en garde ses lecteurs : «on ne saurait trop recommander aux personnes qui étudient les épis [ou écussons] de ne point s'arrêter aux apparences » [TIS 58, p. 47]. Il juge ce système peu efficace expliquant que «Guénon a cru pouvoir établir huit degrés correspondant à des facultés laitières différentes, c'est-à-dire à un produit qui doit aller en diminuant du premier au huitième » [TIS 58, p. 52]. Tisserant relève alors que la classification du marchand de bétail bordelais souffre d'exceptions en trop grand nombre avec de « nombreux signes de la bâtardise » [TIS 58, p. 52].

Tout comme Magne, Tisserant ne condamne cependant pas la technique d'identification elle-même : « il est hors de toute contestation (...) qu'un écusson de forme régulière, très développé, annonce dans une vache des dispositions marquées à produire du lait... » [TIS 58, p. 53]. En revanche, il rejette la mise en système proposée par Guénon : « (...) Guénon et les partisans absolu de son système ont voulu (...) préciser mathématiquement le rendement en lait correspondant à chaque forme, ils sont allés trop loin et se sont exposés à tomber dans de fréquentes erreurs d'application » [TIS 58, p. 54]. Tisserant développe son argumentation en relevant que la production de lait par les vaches dépend de facteurs multiples et complexes comme la digestion, la respiration, ou encore l'hérédité, l'âge, les conditions d'élevage ou climatiques... Et de conclure : «tout cela ne peut ni se résumer, ni se traduire en un signe extérieur » [TIS 58, p. 54].

Ce qui est reproché à Guénon finalement c'est plus sa tendance à présenter sa technique comme universelle et quasi infaillible que sa technique elle-même qui est considérée comme judicieuse par agronomes et vétérinaires, bien que perfectible. Contrairement aux schémas d'innovations les plus 
courants avec une démarche issue du «haut», ingénieurs ou savants, et des réticences venues du «bas », des praticiens, le cas de la technique Guénon est différent. L'innovation vient du «bas », un marchand de bestiaux, et les réticences et les résistances viennent d'en «haut », agronomes et vétérinaires. Lorsqu'on étudie les processus d'innovation, il est souvent difficile de connaître les mécanismes de refus car ils sont souvent le fait de praticiens anonymes éloignés de la production écrite [DIE 03]. Or, dans ce cas précis, les doutes et résistances des milieux savants sont connus car publiés dans des revues ou bulletins des sociétés savantes agricoles.

Face aux critiques savantes, Guénon choisit de répondre et de défendre sa technique dans les rééditions de son Traité des vaches laitières. Parallèlement, d'autres empiriques continuent à utiliser sa technique et à la juger efficace.

\subsection{Guénon se défend tandis que les empiriques sont toujours séduits}

Les remarques critiques n'entament en rien le recours au système Guénon par de nombreux éleveurs et le concepteur de la technique continue à en faire la publicité dans les rééditions de son Traité des vaches laitières.

Dans la quatrième édition de son ouvrage, dont il précise qu'il a été «considérablement augmentée » [GUE 61], Guénon revendique sa démarche empirique et met en avant l'importance de la pratique, estimant que les remarques critiques des vétérinaires et agronomes ne sont que des points de vue théoriques qui ne «sont point [essentiels] au cultivateur... » [GUE 61, p. V]. Face aux critiques et réserves des savants, agronomes ou vétérinaires, Guénon revendique, encore et toujours, sa qualité de «praticien », ainsi qu'il le mentionne sur la page de titre de la quatrième édition de son Traité [GUE 61]. Il insiste sur son statut d'empirique et s'oppose aux discours savants notamment ceux des vétérinaires. Ces derniers, en revanche, ont affirmé, depuis le début du XIX ${ }^{\mathrm{e}}$ siècle, leur discipline en opposition aux empiriques [HUB 99]. Dans ce cas précis, les vétérinaires, positivistes, affirment leur autorité scientifique en expliquant que l'identification d'une bonne laitière ne peut s'effectuer qu'en tenant compte de l'animal dans son ensemble et dans sa complexité. L'empirique Guénon, de son côté, propose une méthode simple accessible à tous les paysans et éleveurs tout en arguant de son infaillibilité.

Ainsi, certains auteurs conservent-ils la taxonomie proposée par Guénon. En 1862, M. Collot, qui se désigne sur la page de titre de son ouvrage comme «propriétaire-agronome », choisit même d'appeler la technique d'identification des écussons « signe Guénon » [COL 62].

À la fin du $\mathrm{XIX}^{\mathrm{e}}$ et au début du $\mathrm{XX}^{\mathrm{e}}$ siècle, la zootechnie fait face à un paradoxe : la technique Guénon est décriée alors même qu'elle est toujours utilisée voire transmise dans un cadre scolaire aux futurs éleveurs et fromagers.

\section{Paradoxe : persistance des critiques et persistance de la transmission de la technique Guénon}

\subsection{Une technique de plus en plus oubliée à la fin du XIXe siècle}

Durant le dernier tiers du $\mathrm{XIX}^{\mathrm{e}}$ siècle, peu à peu, les références à la technique Guénon se font de plus en plus rares dans les littératures vétérinaire, agronomique et zootechnique. Néanmoins certains auteurs l'évoque encore comme Jules-Edouard Lucas (1880-1965), ingénieur agronome, dans son ouvrage consacré à $L a$ vache laitière, publiée en 1914. Cependant, à ce moment-là, ces références à la technique Guénon ne sont plus que des critiques négatives. Lucas aborde la technique Guénon pour indiquer qu'elle est vaine: «plus la figure était étendue, plus la vache devait fournir de lait. L'expérience ne donne pas la vérification de cette idée séduisante » [LUC 14]. Il ajoute aussitôt que «les promoteurs de cette méthode s'en sont vite aperçus. C'est pourquoi ils ont classé ces figures en neuf groupes, chacun des écussons n'est comparable à son voisin que s'il fait partie du même groupe. La distinction entre ces neufs types est déjà très difficile, la comparaison l'est encore plus. Cette 
difficulté est la négation même de la méthode » [LUC 14]. Le jugement de Lucas est sévère mais il s'agit de considérations émises en 1914 à propos d'une méthode mise au point durant les années 1830. Le recul de près de 80 ans de travaux zootechniques et vétérinaires permet de contester plus facilement certains aspects de la technique Guénon mais les progrès de ces disciplines n'ont cependant pas fait disparaître pour autant la technique et il en est encore fait mention dans certains travaux scientifiques même très critiques comme ceux de Lucas. La technique Guénon, bien qu'imparfaite, a été au centre de travaux de zootechnie durant les deux derniers tiers du $\mathrm{XIX}^{\mathrm{e}}$ et les premières années du $\mathrm{XX}^{\mathrm{e}}$ siècle [JUS 00].

C'est aussi une illustration des enjeux des controverses dans la structuration disciplinaire de l'agronomie et de la zootechnie et, en partie, de la médecine vétérinaire. Cela montre aussi l'importance des acteurs ordinaires, ceux d'en «bas» dans le fonctionnement des sciences et des techniques, y compris lorsque les techniques innovantes se révèlent inopérantes ou erronées. Le cas Guénon est d'autant plus intéressant que, dénigré dans la littérature scientifique, sa technique est toujours enseignée au début du $\mathrm{XX}^{\mathrm{e}}$ siècle dans certaines institutions de formation des futurs fromagers.

\subsection{Une technique toujours enseignée au sein de l'ENIL de Mamirolle (Doubs) au début du $X X^{e}$ siècle}

Le processus d'industrialisation et la mécanisation de la production fromagère réclament de nouveaux gestes techniques et savoir-faire complexes. Ces nouveaux procédés de fabrication s'enseignent à partir des années 1880 dans des institutions spécifiques comme les fruitières-écoles ou les écoles nationales d'industrie laitière (ENIL) à Mamirolle et Poligny (dans les départements du Doubs et du Jura), pour ne prendre que l'exemple de la France de l'Est [KNI 20]. Si la question de l'innovation technique est devenue centrale dans le domaine de la laiterie et de la fromagerie, elle l'est d'autant plus dans les institutions de formation technique qui répondent à ces nouveaux besoins de formation.

Au sein des ENIL l'intention est de proposer une formation résolument pratique par apprentissage concret du métier de fromager en faisant pratiquer aux élèves les gestes techniques au quotidien. Or, à Mamirolle, si le souci principal est d'initier les apprentis aux techniques les plus innovantes, au début $\mathrm{du} \mathrm{XX}^{\mathrm{e}}$ siècle, des techniques plus anciennes continuent d'être transmises, comme la technique Guénon pour la sélection des vaches laitières. On trouve à la page 27 du cahier de zootechnie (cahier de 83 pages) de Marcellin Donadieu, élève de l'ENIL de Mamirolle en 1903 [DON 03], mention du système Guénon ainsi qu'à d'autres techniques pour identifier le plus sûrement possible une bonne vache laitière. Le cahier de l'élève Donadieu permet de montrer qu'il est encore questions de ces techniques empiriques dans la formation des futurs fromagers alors même que ces techniques ont peu à peu disparues des ouvrages de zootechnie. On retrouve dans ce domaine la persistance de l'ancien dont les usages côtoient ceux des techniques nouvelles, tel que l'a montré David Edgerton [EDG 13]. La sélection des vaches laitières occupe une grande place dans les enseignements de de zootechnie à l'ENIL de Mamirolle. C'est un point crucial dans le développement des élevages laitiers et dans la recherche de rendements laitiers maximums, tant sur le plan qualitatif que quantitatif. Ce qui explique peut-être pourquoi, nonobstant de sévères critiques et mises en cause, les enseignants n'hésitent pas à aborder des techniques empiriques telles que celle de Guénon.

Pour conclure, nous pouvons donc affirmer que la technique Guénon est un exemple de la complexité des mécanismes de l'innovation technique en agriculture. Et, dans ce cas, comme dans quelques autres à la même époque, la nouveauté technique provient du «bas » pour être, ensuite, acceptée (puis, dans le cas Guénon, rejetée, surtout après 1860) par les sphères savantes. L'histoire originale de la technique d'identification des bonnes vaches laitières proposée par Guénon montre la grande porosité, au $\mathrm{XIX}^{\mathrm{e}}$ siècle, entre les mondes des empiriques ruraux et la sphère savante des agronomes. Eugène Tisserant n'hésite pas à commenter et discuter le travail et les propositions de Guénon. Autre aspect très intéressant de ce cas particulier, sa persistance dans les enseignements techniques au début du $\mathrm{XX}^{\mathrm{e}}$ siècle alors même que la technique semble tombée en désuétude. Les 
processus d'invention et d'innovation techniques sont donc des phénomènes à la circulation aléatoire et aux multiples acteurs. C'est un schéma de diffusion de la nouveauté technique très éloigné de l'approche d'une diffusion linéaire des innovations, de type «tache d'huile », du haut vers le bas [FLI 95 ; HIL 00]. Le modèle «Guénon » de diffusion de l'innovation, si tant est que l'on puisse le qualifier ainsi, est beaucoup plus complexe révélant les interactions et interconnexions, au XIX ${ }^{\mathrm{e}}$ siècle, entre les petites gens de la terre et les mondes savants et académiques.

\section{Bibliographie}

[ANT 16] ANTOINE A. (dir), Agricultural specialization and rural patterns of development, Turnhout, Brepols, 2016.

[BOI 77] BoICHARD J., L'élevage bovin. Ses structures et ses produits en Franche-Comté, Paris, Les Belles Lettres, « Annales littéraires de l'université de Besançon, n²04 », 1977.

[BOU 98] Boulaine J., Legros J.-P., D’O. de Serres à R. Dumont : portraits d'agronomes, Paris, Lavoisier, 1998.

[COL 62] COllot M., Vaches laitières, bøufs et animaux d'attelage, Paris, Librairie Paul Dupont, 1862.

[DEN 98] DENIS B., «Les thèmes zootechniques dans le Journal d'Agriculture Pratique de 1837 à 1856 », dans M.-Cl. AMOURETTI, Fr. Sigaut (dir.), Traditions agronomiques européennes : élaboration et transmission depuis l'Antiquité, 120 ${ }^{\mathrm{e}}$ congrès CTHS, 23-29 octobre 1995, Aix-en-Provence, CTHS éd., 1998, p. 223-237.

[DIE 03] DiEderen P., VAN MeIJL H., Wolters A., BIJAK K., «Innovation adoption in agriculture: innovators, early adopters and laggards », Cahiers Economie et Sociologie Rurales, n 67, 2003, p. 29-50.

[DON 03] DonADIEU M., Cahier de zootechnie, 1903, Archives départementales du Doubs, 1NUM3, http://recherchearchives.doubs.fr/ark:/25993/a0114321123018yIeUX.

[EDG 13] Edgerton D., Quoi de neuf? Du rôle des techniques dans l'histoire globale, Paris, Le Seuil, 2013 (1 ${ }^{\mathrm{er}}$ éd. Anglaise, 2006).

[FLI 95] FLICHY P., L'innovation technique. Récents développement en sciences sociales. Vers une nouvelle théorie de l'innovation, Paris, La Découverte, 1995.

[GAS 43] GASPARIN A. de, Cours d'agriculture, Paris, La maison rustique, 1843, 6 tomes.

[GUE 38] GUENON Fr., Traité des vaches laitières, Bordeaux, Impr. de Balarac jeune, 1838.

[GUE 61] GUENON Fr., Traité des vaches laitières et de l'espèce bovine en général, Paris, Impr. Remquet, Goupy et $\mathrm{C}^{\mathrm{ie}}$, 1861 ( $4^{\mathrm{e} e ́ d}$.).

[HIL 00] HILAIRE-PEREZ L., L'invention technique au siècle des Lumières, Paris, Albin Michel, 2000.

[HUB 99] HUBSCHER R., Les maîtres de bêtes. Les vétérinaires dans la société française (XVIII ${ }^{e}-X X^{e}$ siècle), Paris, Odile Jacob, 1999.

[JUS 00] JUSSIAU R., MonTMEAS L., « La zootechnie comme discipline d'enseignement, 1840-1950 », dans M. BOULET (dir.), Les enjeux de la formation des acteurs de l'agriculture, 1760-1945, Dijon, Educagri éd., 2000, p. 83-89.

[KNI 20] KNITTEL F., Agronomie et techniques laitières. Le cas des fruitières de l'Arc jurassien (1790-1914), Paris, Classiques Garnier, coll. « Histoire des techniques », 2020 (à paraître à l'automne).

[LAT 79] Latour B., Woolgar S., La vie de laboratoire, la production des faits scientifiques, Paris, La Découverte, 1988 ( $1^{\mathrm{er}}$ éd. 1979).

[LIJ 06] LijouR B., BlanCOU J., «Rapport de Jean-Henry [sic.] Magne sur les progrès de la médecine vétérinaire de 1842 à 1867 », Bulletin de la Société française d'histoire de la médecine et des sciences vétérinaires, 2006-6, p. 6478.

[LOD 56] LODIEU J., Vaches laitières. Etude complète des caractères à l'aide desquels on peut reconnaitre facilement une bonne laitière, Paris, Librairie Masson, 1856.

[LUC 14] LUCAS J.-E., La vache laitière. Hygiène et production du lait, Paris, Librairie agricole de la maison rustique, 1914.

[MAD 06] MAdeline Ph., Moriceau J.-M. (dir.), Acteurs et espaces de l'élevage (XVII ${ }^{e}$-XXI siècles). Evolution, structuration, spécialisation, Caen/Rennes, AHSR/PUR, BHR n, 2006.

[MAG 57] MAGNE H., Choix des vaches laitières, ou description de tous les signes à l'aide desquels on peut apprécier les qualités lactifères des vaches, Paris, éd. La Maison rustique, 1853, $2^{\mathrm{e}}$ éd. 1857. 
[ROC 78] Roche D., Le siècle des Lumières en province. Académies et académiciens provinciaux, 1680-1789, Paris/ La Haye, éd. EHESS/ Mouton, 1978, 2 vol.

[RUT 04] RÜTTEN Th., «Empirisme », dans LECOURT D. (dir.), Dictionnaire de la pensée médicale, Paris, PUF, 2004, p. $407-413$

[THI 95] THIERRY E., Les vaches laitières, Paris, J.-P. Baillières et fils, 1895.

[TIS 58] TiSSERANT E., Guide des propriétaires et des cultivateurs dans le choix, l'entretien et la multiplication des vaches laitières, Lyon, Savy éd., 1858.

[VAL 15] VALLAT Fr., "Vétérinaires et vaches contagieuses, trois siècles de lutte sanitaire française, $\mathrm{XVIII}^{\mathrm{e}}-\mathrm{XX}^{\mathrm{e}}$ siècle », Ethnozootechnie, n 99,2015 , p. 99-105.

[VIS 02] VISSAC B., Les vaches de la République, Paris, INRA éd., 2002.

[VIV 15] VIVIER N., «Le rôle des comices dans le développement de l'élevage bovin (1835-1880)», Ethnozootechnie, $\mathrm{n}^{\circ} 99,2015$, p. $123-130$. 